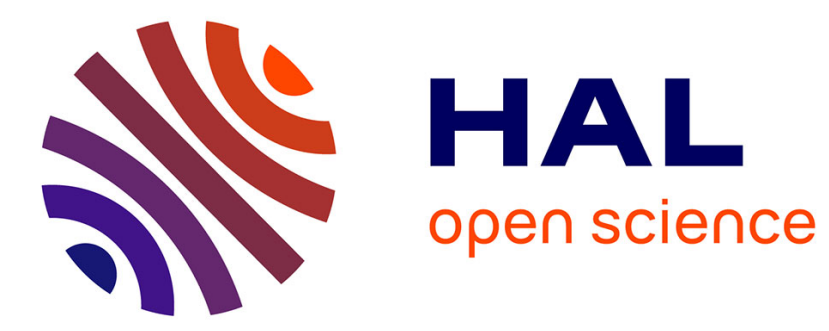

\title{
Un autre regard sur les rapports de développement durable
}

Nathalie Aubourg, Béatrice Canel-Depitre, Corinne Renault-Tesson

\section{To cite this version:}

Nathalie Aubourg, Béatrice Canel-Depitre, Corinne Renault-Tesson. Un autre regard sur les rapports de développement durable. La Revue des Sciences de Gestion, 2011. hal-02332367

HAL Id: hal-02332367

https://hal-normandie-univ.archives-ouvertes.fr/hal-02332367

Submitted on 24 Oct 2019

HAL is a multi-disciplinary open access archive for the deposit and dissemination of scientific research documents, whether they are published or not. The documents may come from teaching and research institutions in France or abroad, or from public or private research centers.
L'archive ouverte pluridisciplinaire HAL, est destinée au dépôt et à la diffusion de documents scientifiques de niveau recherche, publiés ou non, émanant des établissements d'enseignement et de recherche français ou étrangers, des laboratoires publics ou privés. 
La Revue des Sciences de Gestion, Direction et Gestion n 247-248- Organisation

\section{Un autre regard sur les rapports de développement durable}

par Nathalie Aubourg, Béatrice Canel-Depitre, Corinne Renault-Tesson

\section{Nathalie AUBOURG}

Maître de Conférences en sciences de gestion

Laboratoire CERENE - Université Le Havre

France

\section{Béatrice CANEL-DEPITRE}

Maître de Conférences HDR en sciences de gestion

Laboratoire CERENE - Université Le Havre

France

\section{Corinne RENAULT-TESSON}

Maître de Conférences en sciences de gestion

Laboratoire CERENE - Université Le Havre

France 
Résumé :

\section{Un autre regard sur les rapports de développement durable} par Nathalie Aubourg, Béatrice Canel-Depitre, Corinne Renault-Tesson

Après des débuts difficiles, la loi NRE (loi sur les nouvelles régulations économiques) semble maintenant bien acceptée par les grandes entreprises. Les informations concernant les activités environnementales et sociales sont facilement accessibles mais il est difficile d'y voir clair. À partir des rapports de développement durable de trois groupes français, nous tenterons de «tirer leur portrait». Cette recherche s'intéresse à la question de leur volonté de faire converger leurs responsabilités économiques, légales et éthiques. Pour ce faire, le modèle de Carroll, référent de l'analyse de la RSE, nous aidera à la compréhension des niveaux d'action de trois entreprises en matière de RSE. Dans tous les cas, la formalisation de la convergence des responsabilités sous forme de portraits donne des rendus délicats et différents.

Mots-clés : Responsabilité Sociale d'Entreprise, Rapport de Développement Durable, portrait, niveaux de responsabilité

\section{Abstract : \\ Another glance on the sustainable development reports by Nathalie Aubourg, Béatrice Canel-Depitre, Corinne Renault-Tesson}

After a difficult beginning, the French law on New Economic Regulations is now wellaccepted by big companies. Although the social and environmental data are easy to collect, it is still difficult to assess these activities due to the amount of information available.

From the sustainable development reports, we will try to draw the picture of three French companies. The aim of this research is to evaluate whether the economic, legal and ethical responsibilities are met in a balanced way. The idea is to use Carroll's Three-Domain Model as a framework for the development of CSR "Pictures". In any case, formalizing the convergence of responsibilities through pictures provides delicate and different returns.

Keywords : Corporate Social Responsibility, Sustainable Development Report, Picture, Levels of responsibility

\section{Resumen:}

Otra manera de contemplar los informes empresariales sobre el desarrollo duradero por Nathalie Aubourg, Béatrice Canel-Depitre, Corinne Renault-Tesson

Tras principios difíciles, la Ley NRE (Nuevas Regulaciones Económicas ) parece ser aceptada por las grandes empresas. Es fácil de acceder a las informaciones sobre las actividades medioambientales y sociales pero es difícil de comprender e identificar su verdadero posicionamiento. A partir de sus informes sobre el desarrollo duradero, intentaremos sacar una fotografía de tres grupos franceses.

Este trabajo de investigación plantea el problema de su voluntad de hacer converger sus responsabilidades económicas, legales y éticas. El modelo de Carroll, referente del análisis de la RSE, nos ayudará a comprender los niveles de acción de empresas en términos de RSE. En todos los casos, la formalización de la convergencia de las responsabilidades bajo la forma de retratos nos dará resultados matizados y diferentes.

Palabras claves : responsabilidad social de empresa, relación de desarrollo duradero, retrato, niveles de responsabilidad 


\section{Un autre regard sur les rapports de développement durable}

Après des débuts difficiles, la loi NRE (loi sur les nouvelles régulations économiques) ${ }^{1}$ semble maintenant bien acceptée par les plus grandes entreprises. Les informations concernant les activités environnementales et sociales des grandes entreprises sont facilement accessibles mais il est souvent difficile d'y voir clair. Des difficultés majeures d'appréhension du reporting RSE (Responsabilité Sociale d'Entreprise) persistent: la quantité des informations diffusées, le manque de comparabilité d'une année à l'autre, les informations publiées qui s'évertuent à construire une image positive de l'organisation.

Notre problématique consiste à «tirer le portrait » de trois groupes français qui «prennent la pose » dans leur rapport de développement durable. Notre ambition n'est pas d'évaluer leur RSE mais de percevoir l'image émise à travers les choix de communication des RDD en matière de sécurité/santé. Cette recherche s'intéresse à la question de la volonté de convergence entre les différentes responsabilités économiques, légales et éthiques. Pour ce faire, le modèle d'A.B. Carroll, référent de l'analyse de la RSE, nous aidera à la compréhension des niveaux d'action de nos trois entreprises en matière de RSE. Avec l'enrichissement de son modèle en 2003, encore peu répandue au sein de la communauté scientifique, le niveau philanthropique sera fondu dans le niveau éthique et ses sept portraits types nous laisseront espérer une stabilisation de l'expression stratégique de l'entreprise en matière de RSE.

\section{Une approche en trois dimensions de la RSE : vers la définition de portraits-types}

Largement répandue à la fois dans la communauté scientifique et dans les milieux professionnels, la première version du modèle de performance sociale d'A.B. Carroll (1979) interpelle l'entreprise sur la nature de ses responsabilités, les domaines d'application et la forme des réponses apportées. A partir du modèle de performance sociale d'A.B. Carroll, une première représentation de la RSE est suggérée sous forme d'une pyramide. Dans un second

\footnotetext{
${ }^{1}$ Art. 116, loi 2001-420 et décret d'application 2002-221 du 20 février 2002
} 
La Revue des Sciences de Gestion, Direction et Gestion n 247-248- Organisation

temps, afin de rendre compte de l'existence de zones d'intersection, un diagramme de Venn permettra de mettre en lumière sept portraits types de la RSE.

\subsection{Du modèle de performance sociale à la pyramide de la RSE}

Tout en rappelant que les responsabilités fondamentales d'une entreprise demeurent économiques et légales, A.B. Carroll souligne que la société a aujourd'hui des attentes sur le plan éthique. Le niveau discrétionnaire (ou philanthropique) est laissé à la libre appréciation de l'entreprise car contrairement au niveau éthique, il ne répond pas à des attentes clairement exprimées par la société.

Les domaines d'application de la responsabilité sociale sont contingents : ils évoluent en fonction du temps ou du secteur d'activité. Ainsi, les questions de sécurité, de santé, de satisfaction des actionnaires, de discrimination ou de protection de l'environnement n'ont pas toujours connu le même niveau de préoccupation. Cependant, le fait de reconnaître ses obligations morales est une condition nécessaire mais pas suffisante. Encore faut-il envisager des modalités de réponse. A.B. Carroll s'appuie en cela sur la définition de W.C. Frederick (1978) en terme de social responsiveness et envisage quatre stratégies de réponse : réactive (résister le plus possible), défensive (faire seulement le minimum), accomodative (être progressif) ou proactive (être le leader).

La figure suivante illustre ce modèle de performance sociale en 3 dimensions : 
La Revue des Sciences de Gestion, Direction et Gestion n 247-248- Organisation

Figure 1: Modèle de performance sociale
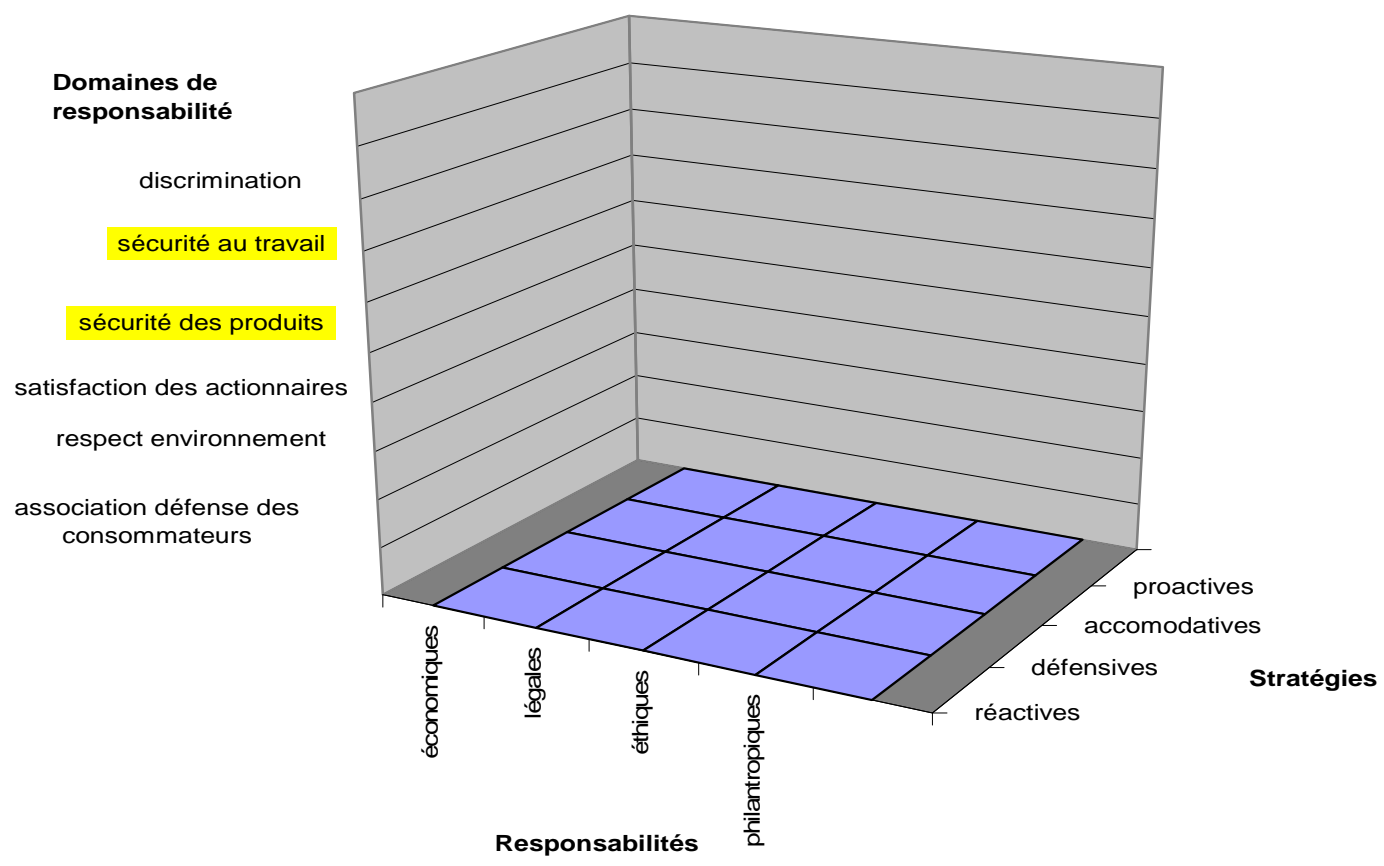

Source: A.B. Carroll, (1979), «A Three-Dimensional Conceptual Model of Corporate Performance », Academy of Management Review, vol 4, n4, p.497-505.

L'intérêt de ce modèle sur un plan académique est de concilier les différentes définitions du concept de responsabilité sociale évoquées dans la littérature. Sur un plan opérationnel, ce modèle a été conçu comme grille d'analyse des différentes dimensions de la performance sociale. Il n'émet pas de recommandations particulières mais doit être compris comme un outil de planification et de diagnostic.

La représentation graphique proposée plus tard (A.B. Carroll ; 1991) sous forme de pyramide des différents niveaux de responsabilité s'est rapidement érigée en «standard» dans la communauté scientifique anglo-saxonne ainsi que dans les milieux professionnels : 


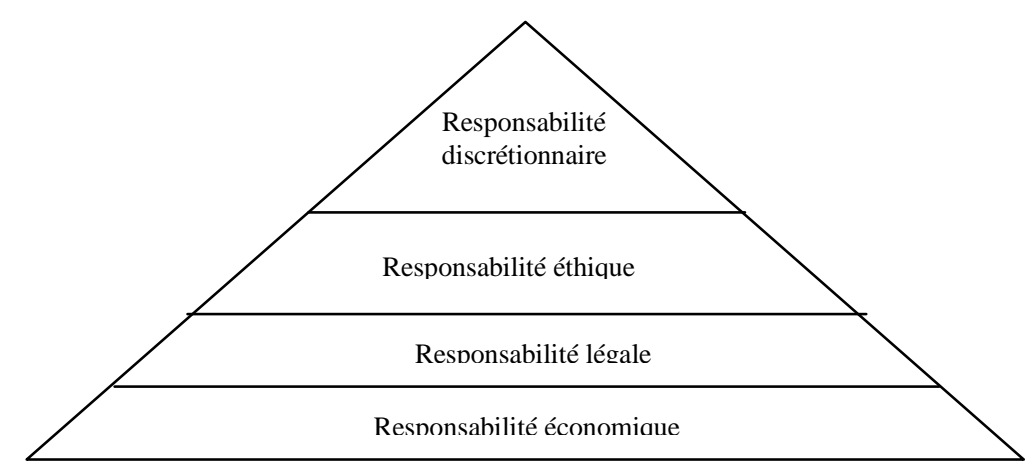

Source: A.B. Carroll, 1991, «The Pyramid of Corporate Social Responsibility: Toward the Moral Management of Organizational Stakeholders", Business Horizons, p. 39-48.

Cependant, en 2003, A.B. Carroll. et M.S. Schwartz ont proposé une nouvelle version de leur modèle qui s'articule autour des points suivants : une remise en cause du choix de la pyramide comme représentation graphique, l'existence de la responsabilité discrétionnaire en tant que telle, une définition plus complète des responsabilités économiques, légales et éthiques.

L'utilisation de la pyramide suggère qu'il y a une hiérarchie dans les niveaux de responsabilité : au sommet de la pyramide, les responsabilités discrétionnaires qui seraient les plus importantes, au bas de la pyramide, les responsabilités économiques représentant le niveau le moins avancé en matière de responsabilité sociale. En fait, il ne s'agissait pas d'effectuer un classement car pour A.B. Carroll, les niveaux économiques et légaux demeurent les plus importants. Par ailleurs, ce schéma ne rend pas suffisamment compte de l'existence de zones d'intersection c'est-à-dire qu'une même entreprise peut dans le même temps satisfaire à des exigences économiques, légales et éthiques.

La distinction d'A.B. Carroll dans son modèle initial entre activité éthique et philanthropique repose sur les attentes supposées de la société : les responsabilités éthiques seraient celles attendues par la société et les activités philanthropiques seraient celles désirées par la société. Or, aujourd'hui, la société attend des entreprises qu'elles mènent des actions philanthropiques. Ainsi, d'une part, si d'un point de vue théorique philanthropie et éthique d'entreprise ne relèvent pas du même ordre ${ }^{2}$, d'un point de vue empirique, les motivations

${ }^{2}$ A. Comte-Sponville, (2004), Le capitalisme est-il moral?, Albin Michel, Paris. 
La Revue des Sciences de Gestion, Direction et Gestion n 247-248- Organisation

philanthropiques sont plus complexes à cerner (K.E Aupperle, 1984). D'autre part, il y a fort à parier que les raisons profondes d'un engagement philanthropique sont de nature économique (B.Shaw and F.R. Post, 1993). Pour toutes ces raisons, le nouveau modèle ne considère plus la responsabilité philanthropique comme une responsabilité à part entière mais comme dépendant des responsabilités économiques et/ou éthiques.

\subsection{De la refonte des responsabilités aux portraits types de la RSE}

M.S. Schwartz et A.B. Carroll proposent une définition beaucoup plus précise des différents niveaux de responsabilité. Ainsi, les responsabilités éthiques et légales sont décrites de façon à rendre compte de motivations très différentes. En effet, on peut faire face à ses obligations légales par soumission, évitement ou anticipation. De la même façon, il est possible d'assumer sa responsabilité éthique par convention, par conscience de la portée de ses actes ou, de façon plus absolue, par déontologie.

Le tableau ci-dessous précise les niveaux de responsabilités et leurs motivations associées :

Tableau 1: La segmentation des niveaux de responsabilités retenus par M.S. Schwartz et A.B.Carroll en 2003

\begin{tabular}{|c|c|c|}
\hline $\begin{array}{l}\text { Niveaux de } \\
\text { responsabilité }\end{array}$ & $\begin{array}{l}\text { Segmentation des } \\
\text { niveaux }\end{array}$ & Motivations \\
\hline \multirow{2}{*}{$\begin{array}{l}\text { Responsabilité } \\
\text { économique }\end{array}$} & Impacts directs & Maximiser le profit et/ou la valeur de l'action \\
\hline & Impacts indirects & Améliorer le moral des salariés ou l'image de l'entreprise \\
\hline \multirow{5}{*}{$\begin{array}{l}\text { Responsabilité } \\
\text { légale }\end{array}$} & Soumission passive & Être conforme à la loi par hasard \\
\hline & $\begin{array}{l}\text { Soumission } \\
\text { restrictive }\end{array}$ & Se soumettre à la loi de façon intentionnelle \\
\hline & $\begin{array}{l}\text { Soumission } \\
\text { opportuniste }\end{array}$ & Tirer avantage des opportunités offertes par la loi \\
\hline & $\begin{array}{l}\text { Évitement des } \\
\text { conflits }\end{array}$ & Éviter des litiges actuels ou futurs. \\
\hline & $\begin{array}{l}\text { Anticipation de la } \\
\text { loi }\end{array}$ & Anticiper l'élaboration d'un texte de loi \\
\hline \multirow{3}{*}{$\begin{array}{l}\text { Responsabilité } \\
\text { éthique }\end{array}$} & Conventionnelle & $\begin{array}{l}\text { Répondre à des normes édictées par la profession ou la société } \\
\text { comme nécessaires }\end{array}$ \\
\hline & Téléologique & Se préoccuper des finalités ou des conséquences de ses actes. \\
\hline & Déontologique & Agir par devoir ou par obligation \\
\hline
\end{tabular}

Cet apport d' A.B. Carroll et de M.S. Schwartz reste, cependant, insuffisant pour permettre de segmenter les niveaux de responsabilité de l'entreprise de façon opérationnelle. En effet, 
selon ces auteurs : un instrument de mesure fiable et valide reste à concevoir. La mise en évidence de l'existence de zones de chevauchement entre les responsabilités mises en lumière par M.S. Schwartz et A.B. Carroll nous permettra de caricaturer nos entreprises.

Ces auteurs proposent une représentation des aspects économiques, légaux et éthiques de la RSE sous la forme d'un diagramme. A partir des zones d'intersection, sept portraits types peuvent être identifiés comme le montre le schéma ci-dessous :

Figure 3: Les sept portraits types de la RSE

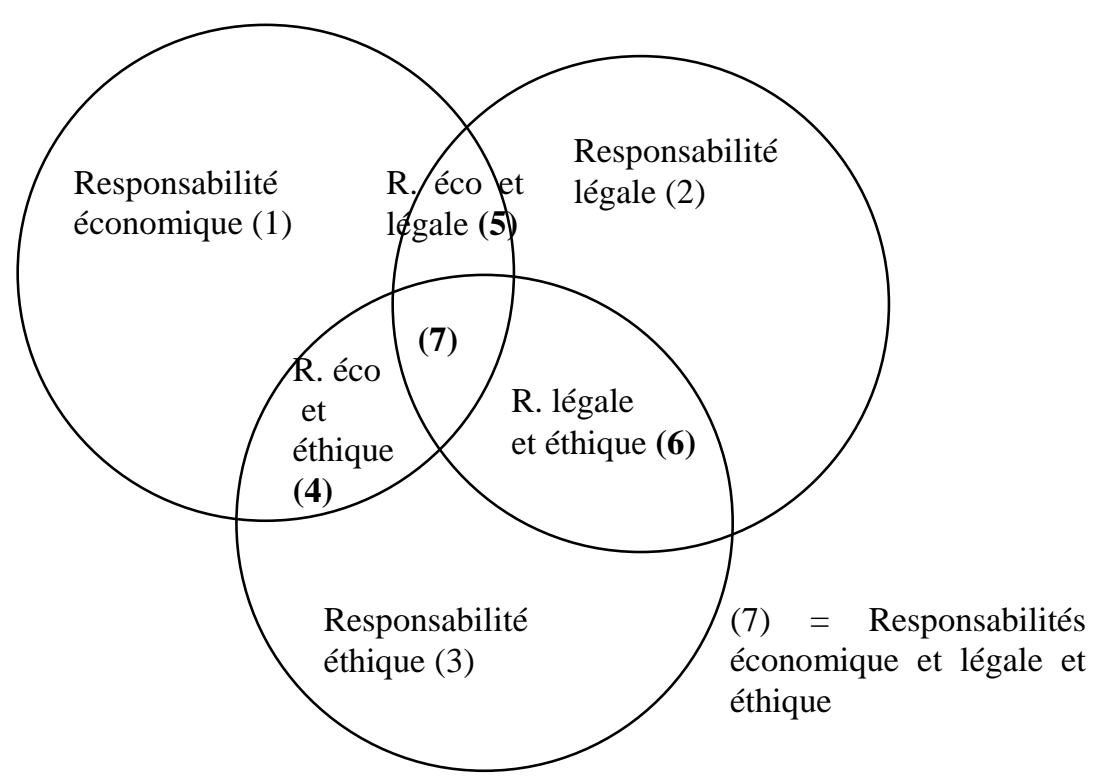

L'idéal est de tendre vers le cœur du modèle (7), c'est-à-dire pour l'entreprise d'assumer à la fois ses responsabilités économiques, légales et éthiques. Cependant, le modèle suggère d'autres situations que ce soit dans les zones d'intersection ou en-dehors de celles-ci.

Ainsi, on peut imaginer des activités purement économiques (1), légales (2) ou éthiques (3). Des décisions peuvent être motivées par des raisons économiques sans tenir compte des considérations légales et/ou éthiques. Il semble plus difficile d'identifier des situations pour lesquelles l'entreprise n'agit qu'en réponse à des engagements légaux ou éthiques (purely legal or purely ethical).

Dans la mesure où la plupart des activités considérées comme légales sont aussi considérées comme éthiques et que les véritables motivations des activités éthiques sont rarement connues, il est peu probable d'observer des situations correspondant au portrait type (6). 
Peu d'activités engagées par les entreprises dans les domaines économique et légal (5) peuvent être qualifiées de non éthiques selon M.S. Schwartz et A.B. Carroll, 2003, p. 517.

C'est pourquoi, ceux-ci envisagent d'observer surtout des situations correspondant aux portraits types (4) et (7).

Au-delà des limites du modèle évoquées ci-dessus, l'étape suivante consiste à représenter, à l'aide de diagrammes de Venn, les priorités en matière de RSE suivant les trois dimensions du modèle pour aboutir à des «portraits ». La taille des cercles est alors proportionnelle à l'importance accordée à chacune des responsabilités.

Ainsi, une représentation de la responsabilité sociale d'une entreprise peut se donner à voir avec des cercles de circonférences différentes car proportionnelles à l'intérêt privilégié par l'entreprise pour l'une ou l'autre des responsabilités.

\section{Tentatives pour « tirer le portrait» de la responsabilité sociale de trois entreprises françaises cotées dans le domaine santé/sécurité}

Cette tâche n'est pas aisée eu égard à l'embarras pour classer une action dans la grille de Carroll. Par ailleurs, la caractérisation des différentes responsabilités, propre aux différents RDD, ajoute une difficulté à réaliser un portrait. Nous avons identifié un processus continu : la même action peut être interprétée différemment dans le temps et la présentation de l'action ne reflète que ce que l'entreprise veut bien montrer. Nous travaillons ainsi comme un artiste qui a du mal à saisir la psychologie de son modèle à partir d'une image qui n'est que partielle et partiale. Après avoir présenté notre méthodologie de travail, nous insisterons sur les difficultés de stabilisation des responsabilités dans un cadre précis.

\subsection{Essai de classement des actions de l'entreprise en niveau de responsabilité}

Afin d'établir des «portraits » de la RSE, nous avons retenu pour notre étude trois entreprises françaises cotées (Air France, Carrefour et Total) évoluant dans des secteurs d'activité très différents (transport aérien, grande distribution et production d'énergie) mais ayant toutes le thème de la santé et de la sécurité comme préoccupation majeure. À partir des RDD de 2006, nous avons fait l'inventaire de toutes les actions entreprises dans le domaine santé/sécurité.

Pour répartir les actions entre les trois types de responsabilité, des codes ont été préétablis et revus en cours d'étude afin que les trois chercheurs aient en tête les mêmes phénomènes au moment du codage (p. 104-108, A.M. Huberman et M.B. Miles, 1991). Afin d'accroître la 
fiabilité de codage, un codage multiple a permis des définitions plus claires et un codage en deux fois a permis d'accroître le degré de cohérence interne. Ensuite le même chercheur ayant codé les différents RDD de «son» entreprise, on peut espérer une moindre variabilité du codage dans le temps pour chacune des entreprises.

Les actions entreprises dans chacune des entreprises ont été réparties entre les trois types de responsabilité retenus par A.B. Carroll.et M.S. Schwartz. L'objectif n'est pas de comparer les trois entreprises entre elles mais de saisir pour chacune d'elle si, en 2006, il est possible de mettre en avant une responsabilité privilégiée.

À partir de la vue panoramique élaborée par les entreprises dans leur RDD, nous nous sommes focalisées sur le domaine de la sécurité/santé. Il nous a été impossible d'aller jusqu'au niveau de précision et de décomposition de chacun des types de responsabilité proposés par A.B. Carroll. et M.S. Schwartz (cf. Tableau n ${ }^{\circ} 1$ ).

La difficulté d'utiliser la segmentation opérée par Schwartz et Carroll peut être traduite à partir d'un exemple, celui de la campagne interne d'intégration des handicapés chez AFKLM. Si nous avons identifié cet exemple comme une responsabilité légale, nous pouvons la classer :

- Comme une soumission passive selon laquelle AF-KLM appliquerait la loi sans autre réflexion

- Comme une soumission restrictive selon laquelle AF-KLM se soucierait de respecter la loi sur l'emploi des handicapés au niveau de $6 \%$ de l'effectif

- Comme une solution afin d'éviter des conflits au sein du personnel sur le manque d'attention en terme de diversité.

De la même façon, quand Total apporte son aide au Ministère de la santé en Angola pour lutter contre l'épidémie de choléra, on peut considérer que Total assume sa responsabilité éthique. Mais est-ce pour des raisons conventionnelles pour répondre aux attentes de la société ou pour des raisons déontologiques en estimant qu'il est de son devoir d'agir ainsi ?

Ne disposant pas d'informations complémentaires et précises, nous n'avons pas utilisé cette segmentation. Un tableau récapitulatif des actions menées par nos trois groupes dans les domaines santé-sécurité va nous permettre de mettre en application la méthode des portraits. 
La Revue des Sciences de Gestion, Direction et Gestion n² 247-248- Organisation

Tableau 2: Grille des actions de RSE dans les domaines sécuritésanté recensées dans les RDD de Air- France - KLM

\begin{tabular}{|c|c|c|}
\hline Responsabilité économique & Responsabilité légale & Responsabilité éthique \\
\hline $\begin{array}{l}\text { Unités « santé et sécurité » et « } \\
\text { mission handicap » } \\
\text { Management QSE }{ }^{3} \\
\text { Partage d'expériences et } \\
\text { harmonisation du traitement des } \\
\text { accidents du travail } \\
\text { Gestion du stress } \\
\text { Certification Servair } \\
\text { Gestion de crise centrale } \\
\text { Comité QSE en interne } \\
\text { Programme de réduction des } \\
\text { nuisances sonores } \\
\text { Guide du management de la sécurité } \\
\text { au travail } \\
\text { Convention annuelle sécurité } \\
\text { Suivi du nombre d'accidents du } \\
\text { travail } \\
\text { Charte de DD basée sur le pacte } \\
\text { mondial des Nations Unies } \\
\text { Formation aux premiers secours à } \\
\text { bord }\end{array}$ & $\begin{array}{l}\text { Programmes de sûreté } \\
\text { Réglementation ATEX }{ }^{4} \text { risques } \\
\text { explosifs } \\
\text { programme saphir } \\
\text { Campagne interne intégration des } \\
\text { handicapés } \\
\text { Certification IOSA }{ }^{5} \text { (politique de } \\
\text { sécurité de l'IATA) } \\
\text { Dialogue avec les associations de } \\
\text { personnes handicapées } \\
\text { Garantie emploi pour salariés avec } \\
\text { handicap } \\
\text { Communication sur missions de } \\
\text { lobbyistes }\end{array}$ & $\begin{array}{l}\text { Assurance santé collective pour tous } \\
\text { les employés } \\
\text { Fondation Air France assistance } \\
\text { enfants } \\
\text { ASF }^{6} \\
\text { Code de déontologie sur la corruption } \\
\text { passive } \\
\text { Bilan de santé } \\
\text { Tests de réductions des nuisances } \\
\text { sonores } \\
\text { Films et informations à bord } \\
\text { Sollicitation passagers pour dons } \\
\text { (argent ou miles) }\end{array}$ \\
\hline
\end{tabular}

\footnotetext{
${ }^{3}$ Qualité Sécurité Environnement

${ }^{4}$ A T E X

${ }^{5}$ IOSA (International Operational Safety Audit) certification délivrée par IATA (International Association Transport Aérien)

${ }^{6}$ Aviation Sans Frontières
} 
Nous souhaitons attirer l'attention du lecteur sur les difficultés de classification des actions à l'intérieur d'un niveau de responsabilité. Par exemple, l'action précitée «sollicitation des passagers pour des dons en argent ou en miles » a été retenue comme appartenant à la responsabilité éthique car il nous a semblé que cette action montre qu'AF-KLM s'intéresse aux conséquences de ses actes pour le bien-être de la société. Il reste possible de comprendre que cette même action puisse relever de la responsabilité économique car encourager les passagers à participer à des projets caritatifs améliore l'image de l'entreprise. Par ailleurs, on peut même aller jusqu'à imaginer que cette forme d'incitation s'inscrit dans un programme plus large de mécénat d'entreprise que la loi encourage, une responsabilité légale peut alors être avancée. Ainsi une même action peut alors appartenir à un ou plusieurs niveaux de responsabilité, c'est certainement le sens que M.S. Schwartz et A.B. Carroll ont voulu montrer dans leur modèle en pointant les zones d'intersection. Nous avons poursuivi la méthode pour l'appliquer à Carrefour.

Tableau 3 : Grille des actions de RSE dans les domaines sécurité-santé recensées dans les RDD de Carrefour

\begin{tabular}{|c|c|c|}
\hline Responsabilité économique & esponsabilité légale & Responsabilité éthique \\
\hline $\begin{array}{l}\text { Déploiement de gammes «nutrition» } \\
\text { des produits à marque propre dans } \\
\text { plusieurs pays } \\
\text { Réduction des teneurs en sel et sucres } \\
\text { des produits à marque propre } \\
\text { Mise en place d'une organisation } \\
\text { globale de gestion de crise } \\
\text { Offre des produits à marque propre } \\
\text { ou 1er prix de meilleur rapport } \\
\text { qualité/prix suite collaboration avec } \\
\text { des nutritionnistes et des } \\
\text { toxicologues } \\
\text { Club fournisseurs «FQ » dans } \\
\text { certains pays }\end{array}$ & $\begin{array}{l}\text { Refonte de la formation Surreté dans } \\
\text { les hypermarchés en France et } \\
\text { compagne de prévention des } \\
\text { accidents du travail en Colombie } \\
\text { Dispositif alerte et de retrait des } \\
\text { produits } \\
\text { Signature d'une déclaration contre } \\
\text { l'insécurité au travail dans le cadre } \\
\text { d'Eurocommerce } \\
\text { Optimisation de l'information } \\
\text { nutritionnelle sur les packagings } \\
\text { 390 audits sociaux dont } 53 \text { ré-audités } \\
\text { Étiquetage nutritionnel sur produits à } \\
\text { marque propre } \\
\text { Signature du code Éthique-Groupe } \\
\text { pour nouveaux embauchés } \\
\text { Réflexion et lancement REACH } \\
\text { Soutien à norme internationale sur la } \\
\text { responsabilité des droits de l'homme } \\
\text { Coopération avec la Commission } \\
\text { européenne et l'OMS pour les bonnes } \\
\text { pratiques des modes de vie sains et } \\
\text { lutter contre l'obésité }\end{array}$ & $\begin{array}{l}\text { Fondation Internationale Carrefour } \\
\text { pour programme Epode «Ensemble } \\
\text { Prévenons l'Obésité Des Enfants » } \\
\text { dans villes pilotes en France, } \\
\text { Belgique et Espagne } \\
\text { Déploiement des semaines de la } \\
\text { nutrition } \\
\text { Programme } \\
\text { professionnelle de collaborateurs } \\
\text { handicapés } \\
\text { Mise en place de hot-lines éthiques } \\
\text { dans } 6 \text { pays sensibles } \\
\text { Ecoute du personnel sur } 11 \text { pays et } \\
21 \text { Business Units en } 2006 \\
\text { Fondation Carrefour pour projets } \\
\text { d'aide humanitaire ou de lutte contre } \\
\text { l'exclusion } \\
\text { Prix de la Croix-Rouge espagnole } \\
\text { remis à la Fondation Carrefour } \\
\text { Solidaridad }\end{array}$ \\
\hline
\end{tabular}


La Revue des Sciences de Gestion, Direction et Gestion n 247-248- Organisation

À travers ces exemples, on remarque que si certaines actions relèvent nettement d'une responsabilité identifiée, d'autres sont à l'intersection de plusieurs responsabilités. Carrefour, en faisant étalage de ses actions de sensibilisation de ses fournisseurs à la sécurité alimentaire, en encourageant les pratiques agricoles durables, en soutenant, à travers la Fondation Internationale Carrefour, le programme Epode «Ensemble Prévenons l'Obésité Des Enfants » envisage-t-elle des impacts économiques, cherche-t-elle à anticiper la loi ou à faire face à des responsabilités éthiques ? Tout dépend de quel point de vue on perçoit les actions énoncées. Les choix opérés en matière de classement font, en tout état de cause, intervenir la subjectivité du chercheur. Ainsi, certaines actions nous laissent devant une difficulté pour trancher son appartenance de manière définitive et nous laisse suggérer des zones d'intersection. Enfin, le cas Total sera également envisagé. 
Tableau 4 : Grille des actions de RSE dans les domaines sécurité-santé recensées dans les RDD de Total

\begin{tabular}{|c|c|c|}
\hline Responsabilité économique & Responsabilité légale & Responsabilité éthique \\
\hline 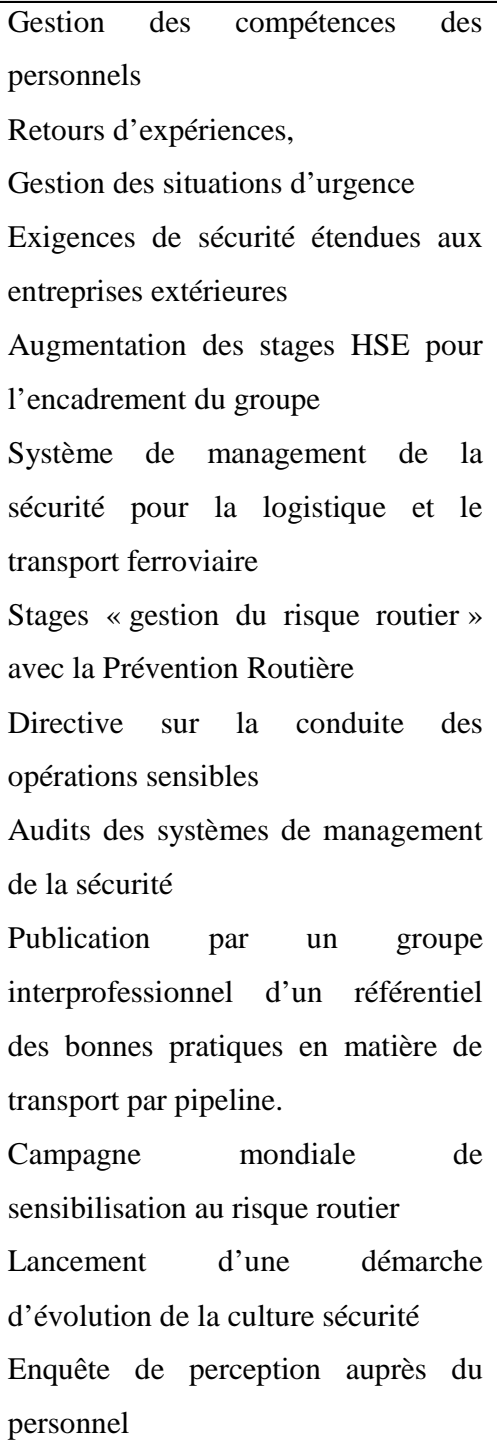 & $\begin{array}{l}\text { Adoption du règlement européen } \\
\text { Reach } \\
\text { Reporting mondial sur les maladies } \\
\text { professionnelles }\end{array}$ & $\begin{array}{l}\text { Poursuite du partenariat avec } \\
\text { l'association Ecomaires } \\
\text { Signature d'une déclaration } \\
\text { d'engagement dans la lutte contre le } \\
\text { sida } \\
\text { Elaboration d'un guide pour lutter } \\
\text { contre le paludisme } \\
\text { Aide au ministère de la santé en } \\
\text { Angola pour lutter contre l'épidémie } \\
\text { de choléra } \\
\text { Mise en place d'un système de veille } \\
\text { face au risque de grippe aviaire } \\
\text { Partenariat avec l'Institut Pasteur }\end{array}$ \\
\hline
\end{tabular}

Nous avons rencontré les mêmes difficultés pour Total que celles rencontrées pour Air France et Carrefour quant à la classification des actions à l'intérieur d'un niveau de responsabilité. Ainsi, le réseau HSE de Total a conduit pour la $3^{\text {ème }}$ année consécutive un reporting mondial sur les maladies professionnelles. L'objectif de ce reporting est d'améliorer les techniques de prévention des risques sanitaires au poste de travail. Cette action a été classée comme responsabilité légale dans la mesure où elle consiste à éviter des litiges actuels ou futurs. On pourrait tout aussi bien penser que cela relève de la responsabilité économique de l'entreprise car cette action peut avoir des retombées en terme d'image ou de la responsabilité éthique car cela montre que l'entreprise se préoccupe des conséquences de ses actes. 
La Revue des Sciences de Gestion, Direction et Gestion n 247-248- Organisation

Malgré ces limites méthodologiques et afin de passer des RDD aux profils d'entreprises en matière de RSE, nous avons procédé au comptage des actions entreprises dans le domaine sécurité-santé pour chacune des responsabilités. Ce sont ces résultats que nous présentons dans le paragraphe suivant.

\subsection{Les difficultés de stabilisation des responsabilités dans un cadre précis}

Notre représentation de la RSE s'avère très partielle mais le recours à d'autres variables plus significatives - comme les budgets alloués, la durée ou la récurrence des actions dans le temps - n'a pas été possible compte tenu de la nature des informations disponibles dans les RDD. Ainsi, les rapports présentent de nombreuses lacunes :

- le manque de données chiffrées systématiques rapportées aux objectifs,

- des informations non financières difficiles à normaliser,

- la grande latitude dont disposent les entreprises pour définir les indicateurs à renseigner,

- les problèmes de pertinence liés à la multiplicité des destinataires.

Dans la mesure où notre démarche n'avait pas pour but d'évaluer l'engagement réel des entreprises en matière de RSE mais de tenter d'en rendre le reporting lisible, nous avons considéré qu'une action ayant une forte occurrence correspondait à ce que l'entreprise voulait montrer de sa RSE.

Après l'affectation des actions à l'un des trois niveaux de responsabilité pertinent, nous avons ensuite mesuré la part relative de chaque type de responsabilité pour chaque entreprise.

\section{Un indicateur d'évolution des responsabilités}

$\rightarrow$ Définition d'un coefficient d'évolution

$\mathrm{C}=\{\mathrm{C} 1, \ldots, \mathrm{Ck}\} \mathrm{k}$ responsabilités

- Soit $\mathrm{P}$ une probabilité a priori sur $\mathrm{C}$,

$\mathrm{Pi}=\mathrm{P}(\mathrm{Ci})->(\mathrm{C}, \mathrm{P})$

C'est-à-dire qu'en l'absence d'information, on considère la probabilité uniforme pour $\mathrm{P}$

$P(C i)=\frac{1}{k}$

- $\quad$ Soit $\mathrm{Q}^{7}$ une autre probabilité sur $\mathrm{C}$

$\mathrm{qi}=\mathrm{Q}(\mathrm{Ci}) \rightarrow(\mathrm{C}, \mathrm{Q})$

\footnotetext{
${ }^{7} \mathrm{Q}$ est une probabilité car la somme des fréquences est égale à 1.
} 
- Le passage de $(\mathrm{C}, \mathrm{P})$ à $(\mathrm{C}, \mathrm{Q})$ est défini par l'importance de la responsabilité Ci lorsqu' on considère la probabilité $\mathrm{Q}$ au lieu de $\mathrm{P}$.

On définit alors un coefficient mesurant cette transformation par :

$$
\alpha i=\frac{q i}{p i}
$$

(Coefficient de passage de (Ci, pi) à (Ci, qi))

On lie un même événement par le rapport des probabilités de la nouvelle situation par rapport à la situation initiale, ici le modèle de référence.

$\rightarrow$ Application du modèle de Carroll

- $\mathrm{C}=\{\mathrm{C} 1, \mathrm{C} 2, \mathrm{C} 3\}$

Les indices 1, 2, 3 correspondent respectivement à la responsabilité économique,

légale et éthique.

Le modèle de référence retenu par A.B. Carroll. et M.S. Schwartz est la probabilité uniforme traduisant l'absence d'information ${ }^{8}$ sur la distribution du modèle.

Chacune des responsabilités a la même probabilité de survenir, on peut donc écrire :

$$
P(C 1)=P(C 2)=P(C 3)=\frac{1}{3}
$$

- L'analyse de l'année 2006 permet d'obtenir les fréquences de réalisation des actions par type de fréquence, Qi. On obtient, par exemple, pour la société Air France-KLM :

$$
q_{1}=Q(C 1)=\frac{19}{29} ; q_{2}==Q(C 2)=\frac{9}{29} ; q_{3}=Q(C 3)=\frac{9}{29}
$$

- La transformation de $(\mathrm{C}, \mathrm{P})$ à $(\mathrm{C}, \mathrm{Q})$ se fait via les coefficients :

$$
\begin{aligned}
& \alpha 1=\frac{q 1}{p 1}=\frac{13 / 29}{1 / 3}=1.34 \\
& \alpha 2=\frac{q^{2}}{p^{2}}=\frac{8 / 29}{1 / 3}=0.83 \\
& \alpha 3=\frac{q 3}{p 3}=\frac{8 / 29}{1 / 3}=0.83
\end{aligned}
$$

Ainsi aci a permis de comparer la situation de 2006 par rapport à une situation initiale uniforme. Il est possible d'envisager ai comme un indicateur de l'évolution du système entre deux dates, ici 2006 et une autre date si nous voulons faire évoluer notre modèle en comparant d'autres données.

Ainsi sont obtenus les coefficients de l'année 2006 pour les trois sociétés cotées :

\footnotetext{
${ }^{8}$ Manque d'information sur la situation initiale -> probabilité uniforme
} 
La Revue des Sciences de Gestion, Direction et Gestion n 247-248- Organisation

Tableau 5 : Actions RSE dans le domaine sécurité-santé recensées en 2006 dans les RDD de trois sociétés cotées

\begin{tabular}{|c|c|c|c|c|c|c|}
\hline & \multicolumn{2}{|c|}{ Air France-KLM } & \multicolumn{2}{c|}{ Carrefour } & \multicolumn{2}{c|}{ Total } \\
\hline & nombre & coefficient & nombre & coefficient & nombre & coefficient \\
\hline $\begin{array}{c}\text { Responsabilité } \\
\text { économique }\end{array}$ & 13 & 1.34 & 5 & 0.69 & 13 & 1.86 \\
\hline $\begin{array}{c}\text { Responsabilité } \\
\text { légale }\end{array}$ & 8 & 0.83 & 10 & 1.36 & 2 & 0.28 \\
\hline $\begin{array}{c}\text { Responsabilité } \\
\text { éthique }\end{array}$ & 8 & 0.83 & 7 & 0.95 & 6 & 0.86 \\
\hline Total des actions & 29 & 3 & 22 & 3 & 21 & 3 \\
\hline
\end{tabular}

Il apparaît que pour chaque entreprise, un type de responsabilité est prééminent, voire même proéminent si l'on veut maintenir un vocabulaire «photographique». C'est à partir de ce dernier tableau que nous allons tenter de figer l'image à l'instant t pour «Tirer le portrait » de nos trois grands groupes.

\section{Les tirages de « portraits » de RSE des trois sociétés}

Nous avons continué notre travail d'approche en souhaitant utiliser les «portraits » proposés par M.S. Schwartz et A.B. Carroll dans leur article de 2003.

Sachant que nous partons, en théorie, d'un diagramme équilibré en trois responsabilités d'égale importance (voir figure 3), toute prédominance d'une responsabilité augmentera proportionnellement la circonférence du cercle correspondant (voir tableau 5). Les actions relevées dans les RDD de nos trois entreprises nous autorisent ainsi à tirer les portraits types suivants : 
La Revue des Sciences de Gestion, Direction et Gestion n ${ }^{\circ} 247-248$ - Organisation

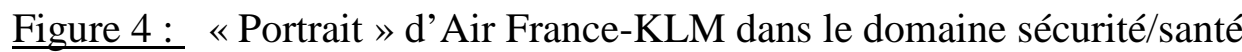

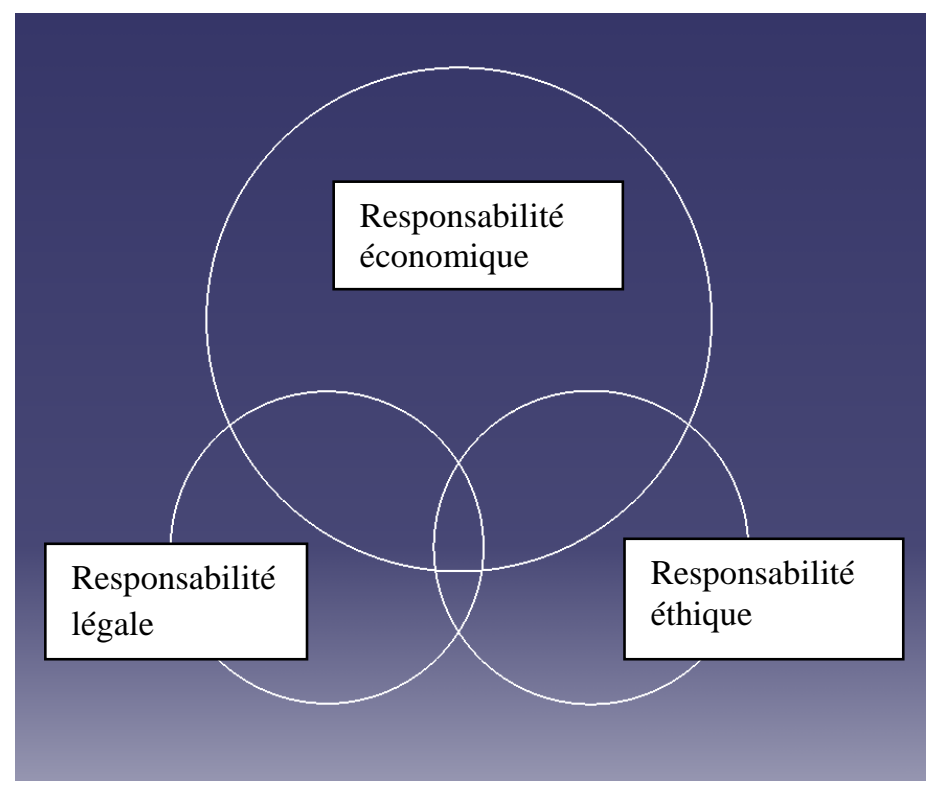

Figure 5: «Portrait » de Carrefour dans le domaine sécurité/santé

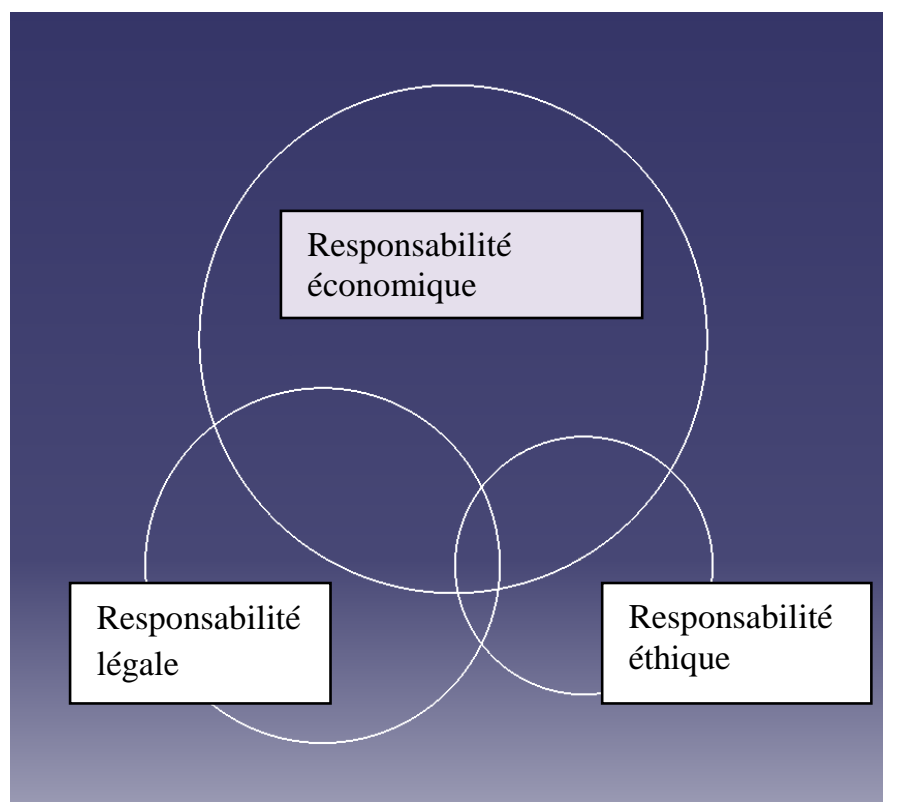


Figure 6: «Portrait » de Total dans le domaine sécurité/santé

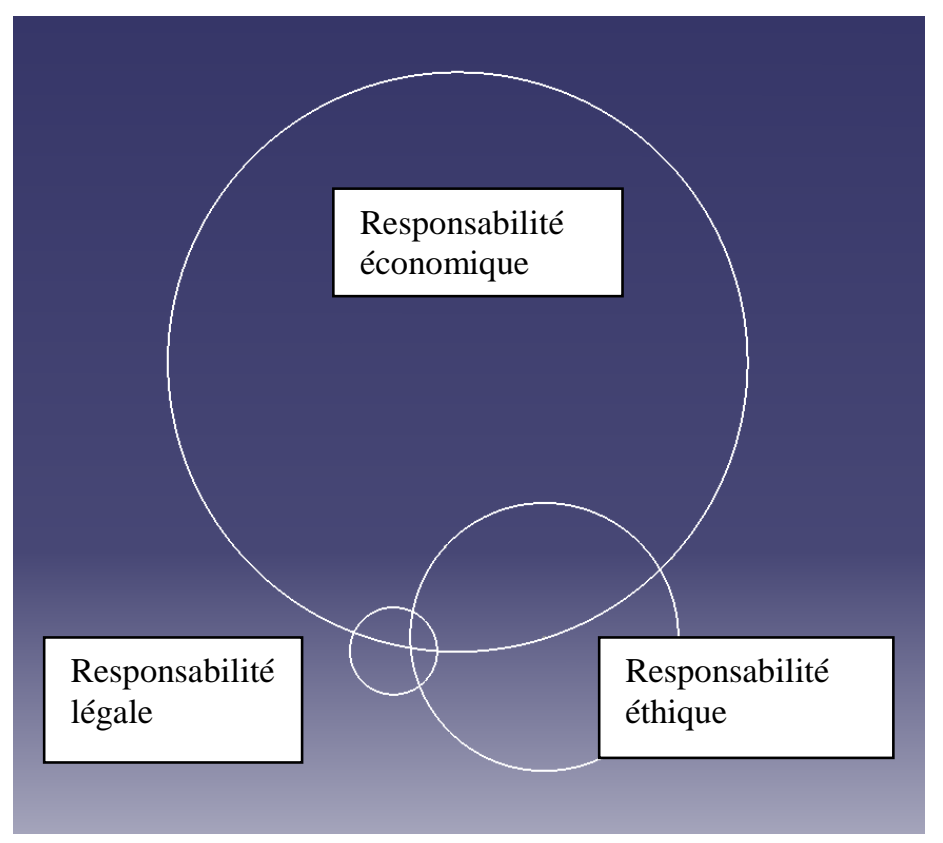

On peut observer des divergences de comportement quant aux responsabilités privilégiées de nos trois grands groupes. Si Carrefour se distingue par son fort penchant pour ses responsabilités légales, Air France-KLM et Total manifestent leur attachement à leurs responsabilités économiques. Ce résultat est, de prime abord, surprenant. Carrefour serait-il donc plus sensible à ses responsabilités légales qu'un groupe comme Total ou Air FranceKLM ? N'oublions pas que nous avons «tiré le portrait» de trois groupes français qui « prennent la pose » dans leur RDD à l'instant t dans le domaine sécurité/santé. Mais, l'on ne peut gommer l'histoire de chacun de nos trois groupes.

De part son métier, Total, soumis à la directive Seveso et au contrôle de la DRIRE, a dû prendre en compte, très tôt, ses responsabilités légales pour répondre aux réglementations et aux contrôles très stricts dont les groupes chimiques et énergétiques font l'objet. On peut donc justement supposer que sa prédilection pour ses responsabilités économiques signifie tout simplement qu'il a totalement intégré, dans l'état actuel de ses obligations, ses responsabilités légales. Ses contraintes réglementaires de sécurité/santé, seraient donc « digérées ».

Pour Air France, suite aux attentats du 11 septembre 2001 aux États-Unis, l'une des premières mesures prises a été de renforcer la sécurité d'une façon drastique (la totalité des bagages passagers doit passer aux rayons $\mathrm{X}$, passage obligatoire aux portiques, utilisation de magnétomètres, fouilles manuelles). Sans oublier que les compagnies aériennes sont 
contrôlées par les États dans tous les pays du monde. Les avions sont certifiés par les États pour avoir le droit de voler, les licences des pilotes sont délivrées par les États, le contrôle aérien dépend des États, l'entretien des avions est soumis à contrôle édicté par les États, les droits du trafic de passagers sont négociés par les États et leur appartiennent. Les responsabilités légales sont totalement «ingérées » par les compagnies aériennes, ces dernières ne peuvent maintenir leurs offres sans respecter ou anticiper les réglementations.

À l'inverse, un groupe comme Carrefour doit faire face aux obligations nouvelles en matière de traçabilité, d'alertes et de retraits de ses produits à risque, de responsabilité face aux agissements de ses fournisseurs, face au problème d'hygiène et de sécurité en magasin, face à la question de l'obésité. Le groupe doit, aujourd'hui, assumer des obligations diverses et apprendre à les intégrer totalement dans ses responsabilités économiques.

«Zoomer » la RSE en matière de sécurité/santé permettrait de percevoir à quel stade d'évolution se situe le groupe. Ainsi, on peut penser qu'il existe un véritable cycle d'évolution des responsabilités RSE en matière de sécurité/santé.

\section{Conclusion : Limites et voies de recherche}

L'intérêt de notre recherche est de tenter d'apporter un éclairage à la lecture des RDD. Nous avons dû démêler les différentes actions pour les transcrire en niveaux de responsabilités. Cette opération nous a permis de peindre «un portrait» de chaque groupe analysé. Ces portraits ne sont qu'un début, à l'avenir, ces portraits de 2006 seront comparés avec les autres portraits que nous continuerons de tirer à partir des éditions futures de RDD. Notre démarche est, certes, séduisante, mais nous ne pouvons en ignorer les lacunes. Malgré l'apport du modèle d'A.B. Carroll., M.S. Schwartz 1 de 2003 à la compréhension de la RSE sous forme de portrait, la difficulté n'en reste pas moins grande :

- L'apport de M.S. Schwartz et A.B. Carroll sur la segmentation des niveaux de responsabilité est sur le fond séduisant mais peut laisser le lecteur perplexe sur sa faisabilité : comment identifier les véritables motivations de l'entreprise ?

- Réaliser des portraits pour clarifier le contenu des RDD est un outil intéressant mais qui en l'état actuel, ne permet pas de rendre compte de zones d'intersection.

- Quel point de vue adopter? Ainsi, A.B. Carroll., M.S. Schwartz reconnaissent que l'internationalisation des entreprises pose problème : quels sont les standards légaux et 
La Revue des Sciences de Gestion, Direction et Gestion n 247-248- Organisation

éthiques à prendre en compte dans le cas d'une multinationale ? Faut-il se placer du point de vue de la maison mère ou de celui de la filiale étrangère ?

- Qu'a voulu réellement montrer l'entreprise à travers son RDD ?

- Peut-on réellement traduire le comportement de l'entreprise à partir des RDD ?

A l'instant $t$, on a pu proposer des portraits de nos trois entreprises qui ne préjugent en rien de leur portrait passé et de leur portrait futur. Dans tous les cas, la formalisation de la convergence des responsabilités sous forme de portraits donne des rendus délicats et différents.

\section{Bibliographie :}

Alberola E., Richez-Battesti N., (2005), « De la responsabilité sociétale des entreprises : Évaluation du degré d'engagement et d'intégration stratégique Évolution pour les entreprises du CAC 40 entre 2001

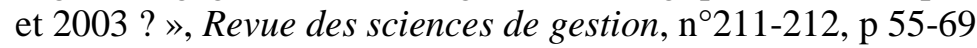

Dontenwill E., (2005), «Comment la théorie des parties prenantes peut-elle permettre d'opérationnaliser le concept de développement durable pour les entreprises ? », Revue des sciences de gestion, $\mathrm{n}^{\circ} 211-212$, p 85-96

Aupperle K.E., (1984), “An empirical measure of corporate social orientation", Research in CSR and policy, vol 6, p.27-54.

Capron M., Quairel-Lanoizelé F., (2004), Mythes et réalités de l'entreprise responsable,

La Découverte, Paris.

Capron M., Quairel-Lanoizelé F., (2007), La responsabilité sociale d'entreprise, La Découverte, Paris.

Carroll A.B., (1979), "A three-dimensional conceptual model of corporate performance", Academy of Management Review, vol.4, n4, p.497-505.

Carroll A.B., (1999), "Corporate social responsibility, evolution of a definitional construct", Business and Society, vol.38, n³, p.268-295.

Carroll A.B., (1991), «The pyramid of corporate social responsability: toward the moral management organizational stakeholders", Business Horizons, p.39-48.

Carroll A.B., Schwartz M.S., (2003), "Corporate social responsability : a three-domain approach", Business Ethics Quaterly, vol.13, n 4, p.503-530.

Comte-Sponville A., (2004), Le capitalisme est-il moral ?, Albin Michel, Paris.

De Combes M., Vandangeon-Dérumez I., (2006), «La Responsabilité Sociale d'Entreprise, un nouveau cadre de pensée et d'action : 15 entreprises en mouvement », colloque de l'ISEOR, Lyon.

Frederik W.C., (1978), "From CSR to CSR: The maturing of business-and-society thought". WorkingPaper $n^{\circ} 279$, Graduate School of Business, University of Pittsburgh.

Huberman A.M. et Miles M.B., (1991), Analyse des données qualitatives : recueil de nouvelles méthodes, Ed. De Boeck, Bruxelles.

Igalens J. et Joras M., (2002), La responsabilité sociale de l'entreprise. Comprendre, rédiger le rapport annuel, Editions d'Organisation, Paris.

Igalens J., (2007), « Étude de la notation sociétale - influence des systèmes économiques et sociaux », congrès de l'AIMS, Montréal. 
La Revue des Sciences de Gestion, Direction et Gestion n 247-248- Organisation

Jonas H., (1990), Le Principe de responsabilité, une éthique pour la civilisation technologique, Ed. Cerf, Paris.

Melkonian T., (2006), «Les cadres supérieurs et dirigeants face au changement imposé : le rôle de l'exemplarité», congrès de l'AIMS, Annecy.

MelkonianT., Monin P., Noorderhaven N., Rouzies A. et Timmers A., (2006), "Être juste, ou être exemplaire ? La fusion Air France KLM à la loupe », Revue française de gestion $\mathrm{n}^{\circ}$ 164, p.229-252.

Mullenbach-Servayre A., (2007), «L'apport de la théorie des parties prenantes à la modélisation de la responsabilité sociétale des entreprises », Revue des sciences de gestion, ${ }^{\circ}$ 223, p109-120

Shaw B. et Post F.R., (1993), "A moral basis for corporate philanthropy", Journal of Business Ethics, 12, p.745-751. 